\title{
A role for naturally occurring alleles of endoplasmic reticulum aminopeptidases in tumor immunity and cancer pre-disposition
}

\author{
Efstratios Stratikos ${ }^{1}{ }^{*}$, Athanasios Stamogiannos ${ }^{1}$, Efthalia Zervoudi ${ }^{1}$ and Doriana Fruci ${ }^{2}$ \\ National Center for Scientific Research Demokritos, Athens, Greece \\ ${ }^{2}$ Department of Paediatric Haematology/Oncology, IRCCS, Ospedale Pediatrico Bambino Gesù, Rome, Italy
}

Edited by:

Paul Eggleton, Exeter University

Medical School, UK

Reviewed by:

Tim Elliott, University of

Southampton, UK

Edwin Bremer, University Medical

Center Groningen, Netherlands

\section{*Correspondence:}

Efstratios Stratikos, National Center for Scientific Research Demokritos,

Patriarhou Gregoriou and Neapoleos

Street, Agia Paraskevi 15310, Athens,

Greece

e-mail: stratikos@gmail.com,

stratos@rrp.demokritos.gr
Endoplasmic reticulum aminopeptidase 1 and 2 (ERAP1 and ERAP2) are key components on the pathway that generates antigenic epitopes for presentation to cytotoxic T-lymphocytes (CTLs). Coding single nucleotide polymorphisms (SNPs) in these enzymes have been associated with pre-disposition to several major human diseases including inflammatory diseases with autoimmune etiology, viral infections, and virally induced cancer. The function of these enzymes has been demonstrated to affect CTL and natural killer cell responses toward healthy and malignant cells as well as the production of inflammatory cytokines. Recent studies have demonstrated that SNPs in ERAP1 and ERAP2 can affect their ability to generate or destroy antigenic epitopes and define the immunopeptidome. In this review, we examine the potential role of these enzymes and their polymorphic states on the generation of cytotoxic responses toward malignantly transformed cells. Given the current state-of-the-art, it is possible that polymorphic variation in these enzymes may contribute to the individual's pre-disposition to cancer through altered generation or destruction of tumor antigens that can facilitate tumor immune evasion.

Keywords: aminopeptidase, antigen presentation, cancer, SNP, cytotoxic responses, adaptive immunity, innate immunity, polymorphism

\section{PART I: ERAP1/2 AND CANCER}

\section{MECHANISMS USED BY THE IMMUNE SYSTEM TO FIGHT CANCER}

The immune system plays a dual role in cancer, on one hand suppressing tumor growth by eliminating cancer cells or inhibiting their outgrowth, and on the other hand promoting tumor progression by selecting tumor cells able to survive inside an immunocompetent host (1-7). The dynamic process that integrates these opposing functions of the immune system - host protection and tumor progression - is termed "cancer immunoediting" and consists of three phases: (i) elimination, (ii) equilibrium, and (iii) escape.

In the elimination phase, cells of innate and adaptive immunity work together to identify and destroy nascent cancer cells, before they become clinically apparent tumors. If a cancerous cell variant is not eliminated in this phase, it may enter the equilibrium phase and remain in a state of functional dormancy under control of cells and mediators of the adaptive immunity. In this phase, the immune system controls tumor growth but does not eliminate it. Cancer cells that acquire mutations can become: (i) invisible to adaptive immunity, i.e., antigen-loss variants or tumor cells that develop defects in antigen-processing or presentation, (ii) insensitive to immune effector mechanisms, or (iii) induce an immunosuppressive state within the tumor microenvironment, enter the escape phase and grow progressively eventually becoming visible tumors. The achievement of the last phase is indicative of a failure in the adaptive immune cells to provide protection from tumor development due to selection of poorly immunogenic tumor cell variants during the equilibrium phase.

Many different cells belonging to the innate and adaptive immunity play an active role in cancer control, from the earliest stages of transformation to the terminal phase of widespread metastasis. The immune cells most involved in the host protection from development of cancer are natural killer (NK) cells and cytotoxic T-lymphocytes (CTLs).

NK cells provide the first immune defense against infections and tumor transformation through recognition and killing of aberrant cells. Their function is finely tuned by the interaction of activating and inhibitory receptors with their specific ligands expressed on target cells (8). Activating receptors recognize ligands expressed on stressed, infected, or transformed cells, whereas inhibitory receptors, prevent $\mathrm{NK}$ cell activation upon interaction with major histocompatibility complex (MHC) class I molecules expressed on target cells (9-14). The reduced surface expression of MHC class I molecules and acquisition of activating ligands in virally infected and transformed cells make these cells particularly vulnerable to NK cell-mediated killing.

CTLs constantly monitor peptide-MHC (pMHC) class I complexes on the cell surface and eliminate virally infected or transformed cells expressing novel peptides derived from abnormal gene products. The generation of these peptides is central in the regulation of CTL and NK cell responses against altered cells. Aberrant antigenic peptide generation can lead to either immune 
evasion or to immune responses against normal cells, initiating or sustaining autoimmune reactions. Antigenic peptides are generated by the concerted action of multiple components of a biochemical pathway termed MHC class I antigen-processing and presentation.

\section{PATHWAY OF ANTIGEN-PROCESSING AND PRESENTATION}

Antigen generation generally starts with the ubiquitin-proteasome pathway where proteins are tagged for proteolytic destruction by the proteasome although other cytosolic peptidases often play important roles (15). The immune cell variant of the proteasome, called immunoproteasome, is more efficient in generating longer peptides, which are often $\mathrm{N}$-terminally extended, compared to the final antigenic epitopes (16). A fraction of these precursors are transported by a specialized transporter [Transporter Associated with antigen-Processing (TAP)] into the endoplasmic reticulum (ER) for further processing. Inside the ER, the precursors are further trimmed by ER-resident aminopeptidases, ER aminopeptidase 1 (ERAP1) and ER aminopeptidase 2 (ERAP2), which excise the N-terminal extensions generating mature antigenic peptides $(17,18)$. These peptides then bind onto nascent MHC class I molecules with the help of a multi-protein complex termed the peptide loading complex. The pMHC class I complexes are then translocated to the cell surface where they can interact with specialized immune system (19).

\section{ROLE OF ERAP1 AND ERAP2 IN THE GENERATION OF ANTIGENIC EPITOPES}

The key role of ERAP1 and ERAP2 in the generation of antigenic epitopes has been repeatedly demonstrated in several cell lines and mouse models. Reduction of ERAP1 expression by RNA interference results in drastically defective presentation for some antigenic epitopes $(17,18,20,21)$ and enhanced presentation or no effect for others. Based on these studies, it has become apparent that ERAP1 has a complex, multifaceted role on the generation of the immunopeptidome (22-24).

In mice models, the complete loss of ERAAP expression (the mouse homolog of ERAP1) specifically inhibited surface expression of MHC class I, but did not affect the expression of MHC class II molecules (17). The expression of pMHC class I on the cell surface depends on the quantity as well as the quality of the peptide supply. In ERAAP-deficient cells, the reduction of MHC class I molecules was due to faster dissociation of pMHC class I from the cell surface rather than to a slower rate of pMHC class I assemble in ER. As a result, ERAAP is important for generating optimal peptides that yield stable pMHC class I complexes (25). Moreover, Hammer et al. observed that the absence of ERAAP disrupts the pMHC class I repertoire in professional and non-professional antigen-presenting cells. From in vivo studies emerged that ERAP1 plays an important role in immune response to viruses, either enhancing or reducing CTL responses to particular viral epitopes and, thereby, helping establish immunodominance hierarchies (25). Undauntedly, expression of endogenous pMHC class I is essential for the generation and maintenance of the normal CD8 ${ }^{+}$ $\mathrm{T}$ cell responses. Splenocytes from ERAAP-deficient mice display an alternative repertoire of peptides as well as differences in the stability of pMHC class I molecules characterized from a diminished ability to elicit HY-specific $\mathrm{CD}^{+}{ }^{+} \mathrm{T}$ cell responses. Interestingly, immunization of ERAAP-deficient mice with splenocytes from wild-type mice resulted in potent $\mathrm{CD} 8^{+} \mathrm{T}$ cell responses, suggesting that ERAP1 plays an important role in modifying antigenic peptides and, paradoxically, its absence enhances immunogenicity $(25,26)$.

ERAP2, the second aminopeptidase demonstrated to be involved in antigen trimming in the ER, is highly homologous to ERAP1 but has distinct specificity (27-29). ERAP1 and ERAP2 have been suggested to perform antigenic peptide trimming in a coordinated manner by forming a functional heterodimer $(18,30)$. Saveanu et al. performed RNA interference to examine the roles of ERAP1 and ERAP2 in trimming of various precursors of the model HIV env epitope, using two different cell lines. The effect of ERAP2 knockdown on cell-surface MHC class I expression and epitope presentation was similar to that of ERAP1 knockdown, suggesting equivalent functions of the two enzymes in the cells studied. Also the greater effect of the double knockdown in some cases suggests that each enzyme can function independently, so that their effects are additive (18).

Overall, the exact effect of the ERAP1 and ERAP2 activities on antigen presentation can be highly variable and difficult to predict. Any factor that can influence the generation of the immunopeptidome may contribute to this, including the cell line used, the $\mathrm{MHC}$ class I alleles, whether the cell contains immunoproteasomes or constitutive proteasomes, the activities of cytosolic aminopeptidases and the sequence of the epitope studied. Regardless, ERAP1 and ERAP2 are undoubtedly important factors that influence the generation of the immunopeptidome, with ERAP1 having a dominant role $(18,22,25)$.

\section{ERAP1 IN INNATE IMMUNITY}

ERAP1 has been found to play important roles in innate immune responses. Namely, ERAP1 has been involved in the shedding of cytokine receptors including the type I TNF receptor (TNFR1), type I IL-6 receptor (IL-6Ra), and type II IL-II decoy receptor (31-33). Additionally, macrophages were found to produce a secreted form of ERAP1 in response to interferon- $\gamma$ and liposaccharides through a TLR-mediated mechanism that leads to enhanced phagocytosis $(34,35)$. Similarly, human PBMCs exposed to ERAP1 externally are activated and show enhanced production of cytokines and chemokines, through mechanisms involving the NLRP3 inflammasome (Aldhamen et al. J Innate Immunity, in press). This secreted form of ERAP1 was found to be enzymatically identical to the ER-retained form and only differ in glycosylation patterns consistent with maturation through the secretory pathway. Since ERAP1 does not contain an ER retention signal it has been hypothesized that it is normally retained inside the ER through interactions with specific ER-resident proteins and can be secreted when these interactions are saturated or disrupted (34).

The function of ERAP1 in regulating antigen presentation can also lead to altered NK cell immune responses. ERAP1 knock-out mice exhibit exaggerated innate immune responses early during pathogen recognition and show increased activation of NK and NKT cells and production of inflammatory cytokines (36). ERAP1 silencing in T cell lymphoma RMA results in tumor rejection in syngeneic mice by triggering NK cells and subsequently $\mathrm{T}$ cell 
$\left(\mathrm{CD}^{+}{ }^{+}\right.$and $\left.\mathrm{CD}^{+}\right)$anti-tumor responses. This rejection does not depend on a simple quantitative reduction in surface MHC class I expression, but is rather the result of changes in the MHC class I-peptide repertoire, because replacement of endogenous peptides with high-affinity mature antigenic peptides was sufficient to rescue the inhibitory activity of NK cells (37). Furthermore, ERAP1 knock-out mice show high frequencies of terminally matured as well as licensed NK cells expressing Ly49C and Ly49I receptors consistent with enhanced NK activation by pro-inflammatory stimuli in those mice (36). Together, these findings suggest an important role for ERAP1 in modulating innate immune responses during the earliest stages of pathogen recognition, a role that may be of particular importance to immune responses toward malignantly transformed cells.

\section{ALTERED LEVELS OF ERAP1 AND ERAP2 CAN FACILITATE TUMOR IMMUNE EVASION}

The enzymatic activity and expression levels of the mouse homolog of ERAP1, ERAAP, have been demonstrated to be key for the immune evasion of tumor cells in two distinct murine models. In one study, the authors showed that ERAP1 downregulation was sufficient to stimulate the cytotoxic activity of NK cells and to result in tumor growth arrest (37). In another study, down-regulation of ERAP1 elicited specific CTL responses against a cryptic tumor-associated antigen that was normally destroyed by ERAP1, resulting in tumor growth arrest and enhanced survival (38). These two studies clearly demonstrated that ERAP1 expression can be critical for immune evasion of solid tumors. Moreover, by using cell lines with two different levels of ERAAP expression levels James et al. established that the induction of anti-tumor immune responses can be titrated based on ERAAP activity, laying the groundwork for the hypothesis described here (38).

In humans, defective expression of components of the antigenprocessing machinery has been associated with the progression and clinical outcome in several types of cancer. The availability of specific ERAP1 and ERAP2 antibodies has allowed researchers to investigate the expression and tissue distribution of these enzymes in a large number of tumor cells of various origins.

In one study, expression of ERAP1 and ERAP2 was detected in all tumor cell lines examined, including melanoma and various type of carcinomas, although at highly variable levels and independently of each other (39). The amount of ERAP1 appears to be more closely coordinated with cell-surface HLA class I molecules, suggesting a secondary involvement of ERAP2 in the generation of ligands for HLA class I molecules at least on quantitative levels. However, this study did not consider that ERAP2 is missing in $25 \%$ of the population (40). Notably, upregulation of ERAP1 and ERAP2 in ERAP-low tumor cells was found to enhance HLA class I surface expression, suggesting that abnormal HLA class I levels in tumor cells may result from defective expression of these enzymes (39).

In another study, a heterogeneous expression of ERAP1 and ERAP2 was detected in a panel of 28 melanoma cell lines (41). A concordant expression between mRNA and proteins for these genes was detected in many cell lines, except four in which an aberrant ERAP2 transcript resulted in total absence of ERAP2 protein expression.

Expression of ERAP1 and ERAP2 was subsequently investigated in 39 different cell types derived from 24 normal non-lymphoid tissues and their malignant counterparts (42). In normal tissues, expression of ERAP1 and ERAP2 was limited to epithelial components. The two enzymes were co-expressed, singly expressed or not expressed, depending on the cell type. HLA class I expression appeared to be independent of ERAP1 and ERAP2 expression and only in nine cell types it was coordinated with both enzymes. In tumor samples, the expression of either or both enzymes was retained, lost, or acquired as compared to the normal counterparts, depending on the tumor histotype. Loss of at least one enzyme was the most frequent phenotype accounting for $86 \%$ tumors. Remarkably, in four types of carcinomas (breast, ovary, liver, and lung carcinomas) arising from normal counterparts co-expressing ERAP1 and ERAP2, none of the 26 tested samples retained this phenotype. ERAP1 was lost in all tested breast, ovary, and lung carcinomas and in 6 out of 7 liver carcinoma samples tested, whereas ERAP2 was retained only in 9 out of 26 samples. The double-negative phenotype was significantly associated with lack of detectable HLA class I molecules. Thus, in vivo transformation affects the expression of ERAP1 and ERAP2, together and individually, leading to losses, gains, or imbalances.

In another study, expression of ERAP1 and ERAP2 was investigated in 300 normal kidney tissues and 334 renal cell carcinoma lesions (43). A heterogeneous and discordant expression of the two enzymes was detected in the different regions of the normal kidney epithelium. In renal cell carcinomas, ERAP1 and ERAP2 appear to have a different behavior, being the first more frequently up-regulated and the latter more frequently down-regulated, as compared to the normal counterpart. None of the clinical parameters investigated was found to be associated with ERAP1 and ERAP2 expression.

ERAP1 expression was also tested in 101 cervical carcinoma patients including adenocarcinomas and squamous cell carcinomas, and correlated with clinical outcome (44). ERAP1 expression was observed in most cases ( 85 out of 101) and overall never totally lost. Partial ERAP1 loss was significantly associated with reduced overall survival and disease free survival. In multivariate analysis, ERAP1 down-regulation was demonstrated to be an independent predictor for worse overall survival and disease free survival, and significantly associated with lymph node metastases.

In another study, ERAP1 expression was examined in 50 esophageal carcinoma lesions and compared with clinicopathological parameters (45). In these tissues, ERAP1 expression was lost or down-regulated in 20 and $28 \%$ of cases, respectively, and significantly associated with the depth of tumor invasion. The authors showed that ERAP1 expression was partially or totally lost in cervical intraepithelial neoplasia and cervical squamous cell carcinoma as compared to normal epithelium of uterine cervix (46), but association with clinical outcome was not investigated.

Taken together, these studies suggest that normal function of ERAP1 and ERAP2 is required for NK cell- and T cellmediated anti-tumor immunity. As a result, the deregulated expression of these enzymes found in tumors may cause improper 
antigen-processing and contribute to escape for host immune surveillance.

\section{PART II: ERAP1/2 SNPS}

\section{ERAP1/2 SNPS AND ASSOCIATION TO HUMAN DISEASE}

Both ERAP1 and ERAP2 are naturally polymorphic and more than a dozen coding single nucleotide polymorphisms (SNPs) in their genes have been associated with pre-disposition to a variety of human diseases primarily of autoimmune etiology (Figure 1; Table 1). Such links are more evident for diseases that are strongly associated with particular HLA class I alleles, implying that the role of ERAP1 and ERAP2 in disease pathogenesis is through the pathway of antigen-processing and presentation. The most prominent example is the association of ERAP1 with Ankylosing Spondylitis (AS), a chronic inflammatory rheumatopathy of the lower spine with an autoimmune etiology. GWAS studies have consistently shown that various ERAP1 coding SNPs are associated with pre-disposition to AS (47-49). The variants that appear to confer risk susceptibility are: rs17482078-C (R725Q), rs30187-T (K528R), rs2287987-T (M349V), rs26653-C (R127P), rs10050860C (D575N), and rs27044-G (Q730E), with each individual one contributing odd ratios of about $1.3-1.4(47,48,50)$. Due to the strong linkage disequilibrium in the ERAP1/2 locus, specific haplotypes have been associated with either pre-disposition or protection against AS. A pre-disposing haplotype has been proposed to comprise of $730 \mathrm{E}, 575 \mathrm{D}$, and $528 \mathrm{~K}$ and a protective one of $528 \mathrm{R}$, 276I, and 127P (51). Another pre-disposing haplotype also carries an ERAP2 SNP (ERAP1 730Q, ERAP1 528K, and ERAP2 392N) (52). Evans et al. showed that ERAP1 contribution to AS could be primarily attributed to rs30187-T (528K), with secondary effects found for rs10050860-C (575D) and rs17482078-C (725R) (48).

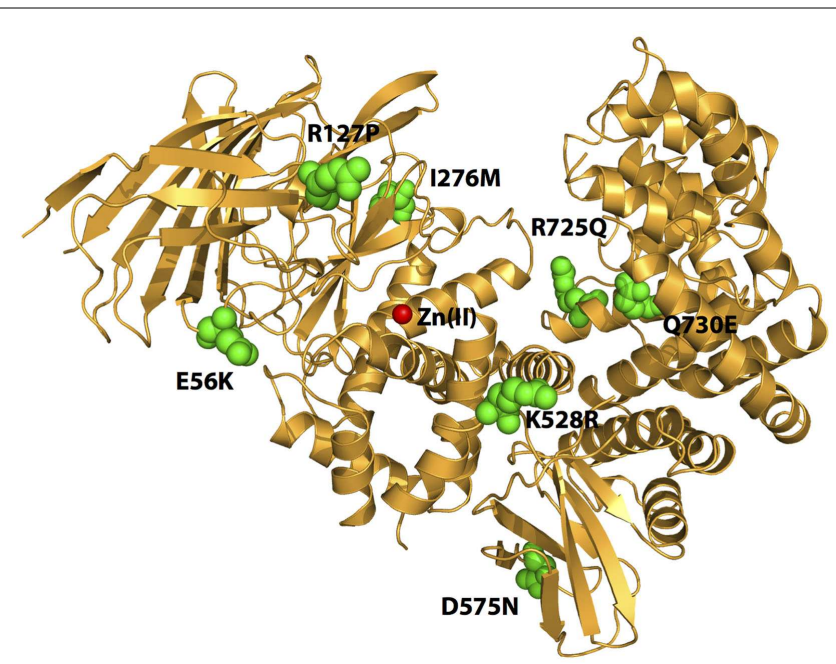

FIGURE 1 | Schematic representation of the crystal structure of ERAP1 (PDB code: 3MDJ). The amino acids at the sites of polymorphic variation that have been found to either affect enzyme function or to associate with pre-disposition to cancer are indicated by green spheres. The catalytic $\mathrm{Zn}$ (II) atom at active site of the enzyme is shown by a red sphere. Note that the polymorphic sites are distal to the active site and distributed throughout the enzyme.
Most notably ERAP1 is associated with AS only in the presence of at least one HLA-B27 allele, which is also the greatest known AS risk factor $(48,49)$ suggesting that the pathogenic effect is mediated through aberrant generation of HLA-B27 ligands either in the context of arthritogenic self-peptides or the generation of pro-inflammatory non-canonical HLA-B27 structures.

The association of ERAP1 with diseases in the context of specific HLA class I alleles is also observed in the cases of Behçet disease (BD) and Psoriasis. A GWAS study in a Turkish population, where $\mathrm{BD}$ is frequently encountered, identified rs10050860-T (575N) and rs17482078-T (725Q) as increased risk variants, with odd ratios significantly higher for HLA-B51 positive individuals (53). Similarly to HLA-B27 and its association to AS, HLA-B51 is the greatest known risk factor for BD. A recent study showed that six positions within the peptide-binding cavity of MHC-I molecules are primarily responsible for the association of particular HLA alleles (including HLA-B51) with BD, implying that aberrant peptide binding by HLA-B51 is central to the pathogenesis of BD (54). Another GWAS study using a population of European ancestry demonstrated that rs30187-T (528K) increases risk for psoriasis, but only in the presence of at least one copy of the allele HLA-Cw*0602 (55). In addition to these well-established cases, ERAP1 SNPs, with rs30187 as a prominent example, have also been associated with other diseases. Specifically, the polymorphism rs30187-C (528R) has been associated with essential hypertension (56) and the rs30187-T (528K) variant with Multiple Sclerosis (57).

ERAP2 has been associated with AS independently of ERAP1. Two functional SNPs have been described: coding rs2549782 (K392N) and rs2248374, a SNP that greatly influences ERAP2 expression (49). Furthermore, another ERAP2 SNP (rs2910686) was associated with AS in HLA-B27-negative cases (49). ERAP1 and ERAP2 SNPs can form haplotypes that may be more relevant to disease pathogenesis (52). The ERAP2 coding SNP rs2549782 $(\mathrm{K} 392 \mathrm{~N})$ is also linked with preeclampsia and with resistance to HIV-1 infection (58-61). The minor allele G is significantly associated with preeclampsia in African-American and Australian populations, but not in a Chilean population $(58,60,61)$. A casecontrol study in a cohort of Italian HIV-exposed seronegative individuals showed that the homozygote GG genotype (encoding Lys/Lys) is overrepresented in the HIV-exposed seronegative sample (59). Recently, Kuiper et al. identified variants near the ERAP2 locus that are associated with birdshot chorioretinopathy (BCR) (62). This GWAS study identified rs7705093-T (OR: 2.3 ), to be in perfect linkage disequilibrium with rs10044354, a variant that affects ERAP2 protein expression levels, with individuals homozygous for the $\mathrm{C}$ allele showing almost none ERAP2 expression. BCR is a useful prototype of disease-HLA association since it exhibits the strongest documented HLA class I association for a human disease ( $>95 \%$ of cases carry the HLA-A29 allele) implicating antigenic peptide processing and presentation in the pathogenesis of this disease.

Overall, a very strong genetic link between ERAP1/2 SNPs and HLA-associated autoimmunity has been established and has contributed to our understanding of the pathogenesis of these diseases. The genetic variability of ERAP1 and ERAP2 appears to constitute part of the natural variability of immune responses 
Table 1 | Most common ERAP1 and ERAP2 SNPs, relation to disease, HLA, and functional consequences.

\begin{tabular}{|c|c|c|c|c|}
\hline & ERAP1/2 SNP & Disease association & HLA class I link & Affects \\
\hline \multirow[t]{7}{*}{ ERAP1 } & rs3734016 (E56K) & HPV-induced cervical carcinoma & & Expression levels \\
\hline & rs26653 (R127P) & Ankylosing spondylitis, HPV-induced cervical carcinoma & & Expression levels \\
\hline & rs2287987 (M349V) & Ankylosing spondylitis & & \\
\hline & rs30187 (K528R) & $\begin{array}{l}\text { Ankylosing spondylitis, psoriasis, essential hypertension, } \\
\text { multiple sclerosis }\end{array}$ & $\mathrm{B}^{*} 27 \mathrm{CW}^{*} 0602$ & Activity and specificity \\
\hline & rs10050860 (D575N) & Ankylosing spondylitis, Behçet disease & & Activity \\
\hline & rs17482078 (R725Q) & Ankylosing Spondylitis, Behçet disease & $B * 51$ & Activity \\
\hline & rs27044 (Q730E) & Ankylosing spondylitis, HPV-induced cervical carcinoma & & Activity and specificity \\
\hline \multirow[t]{2}{*}{ ERAP2 } & rs2549782 (K392N) & $\begin{array}{l}\text { Ankylosing spondylitis, preeclampsia, resistance to HIV } \\
\text { infection }\end{array}$ & & Activity and specificity \\
\hline & rs2248374 (non-coding) & Ankylosing spondylitis & & Expression levels \\
\hline
\end{tabular}

and may therefore contribute to pre-disposition to any number of diseases actively fought by the adaptive immune response.

\section{POLYMORPHIC STATE OF ERAP1/2 AFFECTS ENZYME FUNCTION AND ANTIGEN GENERATION}

The multitude of genetic and population studies linking ERAP1 and ERAP2 SNPs to pre-disposition to autoimmunity and viral infections prompted several research groups to examine the effects of the identified polymorphic variations on the enzyme's biological function and molecular mechanism. To date, most studies have focused on the most well disease-associated SNPs, namely K528R, D575N, R725Q, and Q730E.

The SNP at position 528 has been repeatedly demonstrated to influence ERAP1 enzymatic activity. Various in vitro studies have shown that the 528R variant exhibits less enzymatic activity, compared to the 528K $(48,63-66)$. Cell-based experiments have also demonstrated the importance of that position: HeLa cells transfected with ERAP1 528R and a HLA-B27 peptide precursor displayed a reduced amount of HLA-B27 molecules on the cell surface compared to ERAP1 528K transfectants (64). Furthermore, the presentation of specific MHC class I epitopes was negatively influenced in cells transfected with the 528R variant and their $\mathrm{N}$-extended precursors, as shown by CTL activation assays $(67,68)$.

Studies regarding polymorphic positions Q730E, D575N, and $\mathrm{R} 725 \mathrm{Q}$ have been more complicated to interpret. In vitro assays have suggested that position 730 may or may not have an influence in the enzymatic activity, depending on the substrate used $(63,64)$. Regardless, a cell-surface HLA class I expression assay showed that this SNP could influence the generation of a specific HLA-B27-restricted epitope (64). Some studies showed that position 575 does not influence ERAP1 activity in vitro $(48,63)$, while another study showed that the $575 \mathrm{~N}$ variant exhibits greater activity compared to 575D (66). Regarding position 725, Evans et al., using recombinant enzymes, showed that $725 \mathrm{Q}$ negatively influences enzymatic activity toward a model fluorigenic peptide substrate (48), but an in vitro CTL activation assay did not find any influence of that position to the presentation of an HLA-B27 epitope (68). The complex landscape of functional effects found for these SNPs may be attributed to differences in substrates or assays used, or to differences in background SNPs that are not always consistent between studies. Indeed, in several studies the effects of ERAP1 SNPs have been found to be strongly epitope dependent and to include effects on other mechanistic aspects of ERAP1 peptide trimming, such as substrate inhibition and product activation phenomena $(64,66,69)$.

SNPs in ERAP1 and ERAP2 are often co-inherited as complex haplotypes and may have strong synergism with each other. Unfortunately, most functional studies until now have focused on analyzing the effects of single SNPs and as a result the functional effects of particular disease-associated ERAP1/ERAP2 haplotypes are not always clear especially since the synergisms between individual SNPs are not known. Recently, Seregin et al. analyzed two ERAP1 haplotypes of five SNPs for their effects on HLA-B27 restricted presentation in relation to the pathogenesis of AS and concluded that the high-risk haplotype resulted in reduction in presentation of multiple antigens (70). In another study, Reeves et al. identified nine separate naturally occurring haplotypes in a small population sample based on the five most disease-related SNPs (positions: M349V, K528R, D575N, R725Q, and Q730E) (67). They evaluated the trimming activity of these alleles using a CTL activation assay and showed that each allele exhibits a different activity that is not only dependent on the allele itself but also on the N-terminal extension of the peptide. This study was recently extended to AS patients, showing that ERAP1 haplotypes were clearly stratified in individuals with AS compared to healthy controls and that these functional alleles were poor in generating optimal peptide ligands for HLA-B ${ }^{\star} 2705$ (71). A study focusing on the combined effects of positions 528 and 575 showed that the latter position was dominant in determining enzyme activity (66). Recently, Chen et al. demonstrated that the polymorphic variation in position 730 is critical for rescuing the reduced CTL activation found in the presence of the 528R SNP (68). Overall, ample evidence for strong synergism amongst ERAP1 SNPs are available, although we have very little insight on how these SNPs that are scattered all throughout 
the ERAP1 structure can cooperate to affect the activity of the enzyme.

The influence of ERAP1 polymorphic context has also been studied in the context of the HLA-B27 restricted immunopeptidome and the pathogenesis of AS. Garcia-Medel et al. showed that the presence of ERAP1 with all the AS-pre-disposing polymorphisms (349M, 528K, 575D, 725R, and 730Q) ensured efficient peptide trimming and a higher HLA-B27 stability, compared to the ERAP1 with the AS-protective SNPs (349V, 528R, 575N, 725Q, and 730E) (72). Extending this work, Martin-Esteban et al. showed that synergism between SNPs at positions 528 and 575 can have important effects on cell-surface HLA-B27 presented ligands and more recently Garcia-Medel et al. showed that the particular B27 subtype is critical in determining these effects $(66,73)$. It appears that the interplay between ERAP1 SNPs and HLA class I subtypes is the key to determining the cell-surface immunopeptidome and concomitant cytotoxic responses.

Two ERAP2 SNPs have been shown to have specific effects on the enzyme's biological function: the coding polymorphism rs2549782 (N392K) and the non-coding rs2248374. ERAP2 polymorphism N392K alters the activity and specificity of ERAP2 in a manner much more pronounced compared to the effects described for all coding SNPs of ERAP1 (74). Specifically, in vitro cell-based analysis showed that the $392 \mathrm{~N}$ variant is much more effective in trimming hydrophobic N-terminal residues from antigenic peptide precursors compared to $392 \mathrm{~K}$. In contrast, the rs2248374-G allele can determine ERAP2 expression levels by inducing mRNA instability and non-sense mediated decay (40). The biological consequence of that frequently encountered allele (about 0.5 frequency in six different populations studied) has also been demonstrated; rs2248374-G homozygotes produce no detectable ERAP2 and have reduced levels of MHC class I expression on B-cell surfaces.

Similarly to ERAP1, these ERAP2 SNPs often organize to distinct haplotypes. SNP rs2248374 is in linkage disequilibrium with rs2549782 in various populations studied, thus effectively allowing only the expression of $392 \mathrm{~K}$ variant (40). A noteworthy exception is the Chilean population where the two SNPs are not in linkage disequilibrium, allowing the expression of $392 \mathrm{~N}$ variant (61). Nevertheless, no Chilean genotype was found homozygous for the $392 \mathrm{~N}$ allele, implying a possible negative selection for individuals that are homozygous for that allele. Interestingly, the recent association of ERAP2 expression with pre-disposition to the inflammatory autoimmune disease BCR solidified the idea that ERAP2 polymorphic variation and its effects on antigen-processing may have important repercussions on adaptive immune responses (62).

\section{ERAP1 SNPS AND PRE-DISPOSITION TO VIRALLY INDUCED CANCER}

Genetic variations in genes encoding components of the antigenprocessing pathway have been associated with risk of occurrence malignancy and in particular with cervical carcinoma. Mehta and colleagues analyzed the effect of different ERAP1 SNPs and haplotype combinations on the risk of developing human papillomavirus (HPV)-induced cervical carcinoma.

In the first study, the authors identified two common ERAP1 polymorphisms, R127P and Q730E, significantly associated with increased risk of cervical cancer (75). A haplotype combination consisting of four SNPs, including the minor alleles at R127P and Q730E loci and a major allele at the TAP1 R651C and LMP7 Q145K loci, was significantly associated with a threefold increased cervical carcinoma risk (75). The authors estimated that $12 \%$ of all cervical carcinoma cases were attributable to the occurrence of this haplotype combination (75).

In a subsequent study, the same group investigated which genetic ERAP1 variation affected tumor progression and overall survival in cervical carcinoma patients, and provided the first indication of association of ERAP1 SNPs with ERAP1 protein expression (76). Genotype distributions at the R127P, I276M and K528R were significantly associated with the presence of lymph node metastases. Heterozygosity of E56K and minor allele homozygosity at the R127P loci were significantly associated with decreased overall survival. Multivariate analysis performed on E56K and $\mathrm{R} 127 \mathrm{P}$ genotypes combined with prognostic factors, revealed that the two SNPs loci were not independent predictors of survival. The $\mathrm{R} 127 \mathrm{P}$ variant and the E56K-R127P haplotype were significantly associated with ERAP1 protein expression, with heterozygosity of both individual SNPs and the haplotype consisting of a major allele at the E56K locus and a minor allele at the R127P, being significantly associated with normal ERAP1 expression and better overall survival. Heterozygosity for E56K-R127P haplotype was found to be an independent predictor for overall survival and lymph node metastasis.

The association between ERAP1 SNPs and cervical carcinoma risk or patient survival may be explained by different mechanisms, all related to altered ERAP1 function. Genetic variations at these individual SNPs have been found to affect the expression and stability of transcripts and proteins, to reduce trimming activity or modify substrate specificity $(48,64,76)$. All these effects may affect the ability of HPV to establish persistent infections, but also the ability of transformed cells to evade immune surveillance. The information gathered by these studies has helped to establish a new paradigm on how the polymorphic variation of the adaptive immune system can play a role on both cancer development and prognosis. Although the importance of viral control in the pre-disposition to virally induced cancer is easy to understand, this work has opened the possibility that variable immune responses in the population may also play important roles to cancer pre-disposition.

\section{ERAP1 AND ERAP2 SNPS AND PRE-DISPOSITION TO CANCER - A HYPOTHESIS}

Current knowledge on the roles of ERAP1 and ERAP2 in the human immune response have gradually led to the maturation of the hypothesis that the naturally occurring polymorphic variation in ERAP1 and ERAP2 may play significant roles in the pre-disposition to certain cancers, as well as their progression and prognosis. More specifically, the rational behind this hypothesis is based on the following points:

1. CTL and NK cell responses against malignantly transformed cells are important for eradicating tumor cells often at the early stages of carcinogenesis.

2. Establishment of solid tumors often requires adaptive measures from the transformed cells that lead to the evasion of 
immune responses. Such adaptive measures include changes in antigen-processing and presentation in order to suppress the presentation of tumor-specific antigenic epitopes while also evading NK-cell recognition.

3. Polymorphic variation in ERAP1 and ERAP2, alters their function and their ability to generate antigenic peptides and controls cytotoxic reactions against antigen-presenting cells. This is now well established in autoimmunity and viral infections.

4. ERAP1 and ERAP2 expression levels, which undoubtedly affect effective enzyme activity, are consistently altered in tumors, presumably as an adaptive measure for immune evasion.

5. ERAP1 function can regulate innate immune responses and the production of inflammatory cytokines, a function that can either help eradicate cancer cells or contribute to localize inflammation that can promote tumor growth.

As a result, the polymorphic variability in ERAP1 and ERAP2 may affect the severity of early cytotoxic responses toward transformed cells and influence their chances to accrue genetic adaptations that will allow them to evade the immune system (Figure 2).
Individuals carrying particular ERAP haplotypes in combination with specific HLA class I alleles may therefore be more prone to carcinogenesis, not because of facilitated malignant transformation but because they have an immune system that is less effective in mounting strong cytotoxic responses against cancer cells. Additionally, the function of ERAP1 in regulating inflammatory cytokine production may contribute to these effects by influencing the inflammatory state of the tumor microenvironment.

\section{CONTROLLING ERAP1 AND ERAP2 ACTIVITY FOR TREATING CANCER}

In addition to the possible role of the activity of ERAP1 and ERAP2 on the normal immune control of cancer, a series of recent studies have highlighted that these two enzymes may be important pharmacological targets for boosting immune responses to established cancers. In one study, the genetic down-regulation of ERAP1 in cancer cells that establish solid tumors in mice resulted potent NK cell-mediated cytotoxic responses (37). In another study, the genetic down-regulation of ERAP1 in cancer cells resulted in strong CTL responses against a cryptic tumor antigen normally destroyed by over-active ERAP1 (38). In both studies, tumor

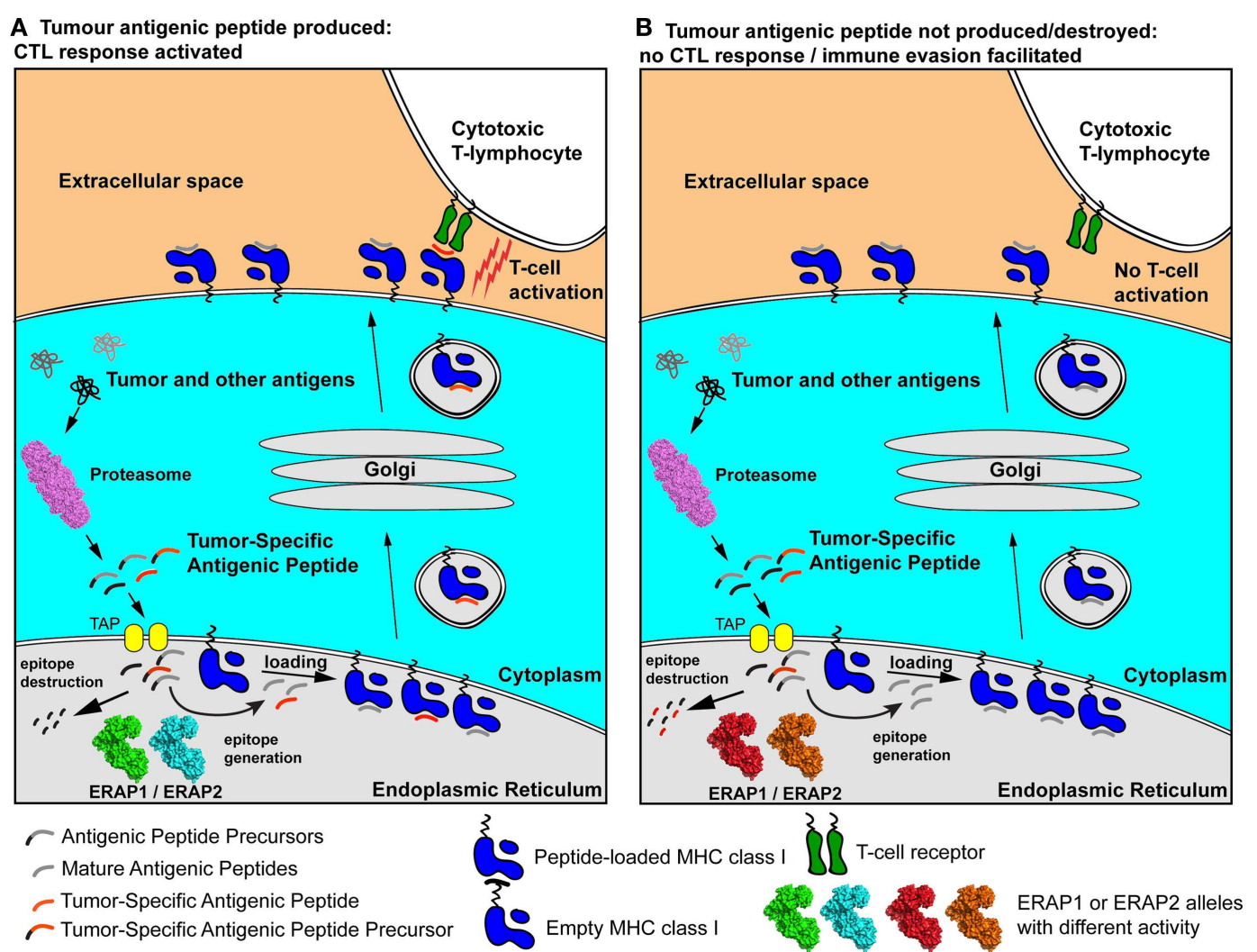

FIGURE 2 | Schematic representation of two extreme scenarios leading to either effective cytotoxic responses against a cancer cell (A) or immune evasion (B). A tumor-specific antigenic epitope or its $\mathrm{N}$-terminal extended precursor is generated by the proteasome and transported into the ER. There, it is further processed by ERAP1/ERAP2 alleles. In case A, the "green" and "cyan" allele effectively generate the mature epitope (red line), which is then loaded onto nascent empty $\mathrm{MHC}$ class I and presented to the cell-surface, activating antigen-specific T cells.
In case B, hyper-active or hypo-active ERAP1/ERAP2 alleles (in red and orange) either fail to produce the mature epitope or destroy it by generating peptides too small to bind onto $\mathrm{MHC}$ class I molecules. As a result, the epitope is not presented on the cell surface and no T cell activation occurs, facilitating immune evasion. The allelic state of ERAP1 and ERAP2 can therefore influence anti-tumor adaptive immune responses pre-disposing individuals to certain cancers by facilitating immune evasion at early stages of malignant development. 
growth was halted resulting in clear therapeutic outcome in mice. In the latter study, the effect was partially reproduced using the small molecular weight metallopeptidase inhibitor Leucinethiol (38). A recently developed potent ERAP1/ERAP2 inhibitor was successfully used to induce CTL responses against the cancer cells used in that study (77). Apart from the obvious extension of these studies conclusions to cancer immunotherapy, one additional conclusion may be drawn: inhibition of ERAP1 activity was not necessarily complete in either study, indicating that even moderate modulation of ERAP1 activity, such as seen in the normal polymorphic variation in this enzyme, may be sufficient to radically affect the potential of cancer immune surveillance.

\section{CONCLUDING REMARKS - FUTURE RESEARCH DIRECTIONS}

In summary, we review the state-of-the-art on the role of ERAP1 and ERAP2 in adaptive and innate immune responses and their role on disease pathogenesis. Combination of knowledge on the role of polymorphic variation on those enzymes and disease pre-disposition with their role in cancer development has led to the formulation of a hypothesis on their direct role on cancer pre-disposition and prognosis. If this hypothesis holds to be true, haplotype analysis of components of the antigen-processing machinery may contribute to our understanding of cancer predisposition and complement other genetic findings on biochemical pathways that control malignant transformation. Because of the genetic heterogeneity of tumors however, it may be difficult to discern such effects at the tumor level and population studies may have to focus on the overall influence of this genetic locus on predisposition to specific cancers. Ongoing and future GWAS studies on cancer patients may yet reveal the importance of immune system variation to developing and fighting cancer, contributing to personalized treatments.

\section{ACKNOWLEDGMENT}

This research was financed by the European Union (European Social Fund) and Greek national funds through the Operational Program "Education and Lifelong Learning" of the National Strategic Reference Framework: Research Funding Program of the General Secretariat for Research \& Technology (Grant ERC-14).

\section{REFERENCES}

1. Dunn GP, Bruce AT, Ikeda H, Old LJ, Schreiber RD. Cancer immunoediting: from immunosurveillance to tumor escape. Nat Immunol (2002) 3:991-8. doi:10.1038/ni1102-991

2. Dunn GP, Old LJ, Schreiber RD. The immunobiology of cancer immunosurveillance and immunoediting. Immunity (2004) 21:137-48. doi:10.1016/j.immuni. 2004.07.017

3. Dunn GP, Old LJ, Schreiber RD. The three Es of cancer immunoediting. Annu Rev Immunol (2004) 22:329-60. doi:10.1146/annurev.immunol.22. 012703.104803

4. Dunn GP, Koebel CM, Schreiber RD. Interferons, immunity and cancer immunoediting. Nat Rev Immunol (2006) 6:836-48. doi:10.1038/nri1961

5. Smyth MJ, Dunn GP, Schreiber RD. Cancer immunosurveillance and immunoediting: the roles of immunity in suppressing tumor development and shaping tumor immunogenicity. Adv Immunol (2006) 90:1-50. doi:10.1016/S00652776(06)90001-7

6. Swann JB, Smyth MJ. Immune surveillance of tumors. J Clin Invest (2007) 117:1137-46. doi:10.1172/JCI31405

7. Crotty S. Follicular helper CD4 T cells (TFH). Annu Rev Immunol (2011) 29:621-63. doi:10.1146/annurev-immunol-031210-101400
8. Raulet DH, Vance RE, McMahon CW. Regulation of the natural killer cell receptor repertoire. Annu Rev Immunol (2001) 19:291-330. doi:10.1146/annurev. immunol.19.1.291

9. Karlhofer FM, Ribaudo RK, Yokoyama WM. MHC class I alloantigen specificity of Ly-49+ IL-2-activated natural killer cells. Nature (1992) 358:66-70. doi:10.1038/358066a0

10. Colonna M, Samaridis J. Cloning of immunoglobulin-superfamily members associated with HLA-C and HLA-B recognition by human natural killer cells. Science (1995) 268:405-8. doi:10.1126/science.7716543

11. Diefenbach A, Jensen ER, Jamieson AM, Raulet DH. Rael and H60 ligands of the NKG2D receptor stimulate tumour immunity. Nature (2001) 413:165-71. doi:10.1038/35093109

12. Kim S, Sunwoo JB, Yang L, Choi T, Song YJ, French AR, et al. HLA alleles determine differences in human natural killer cell responsiveness and potency. Proc Natl Acad Sci U S A (2008) 105:3053-8. doi:10.1073/pnas.0712229105

13. Lanier LL. Evolutionary struggles between NK cells and viruses. Nat Rev Immunol (2008) 8:259-68. doi:10.1038/nri2276

14. Orr MT, Murphy WJ, Lanier LL. 'Unlicensed' natural killer cells dominate the response to cytomegalovirus infection. Nat Immunol (2010) 11:321-7. doi:10.1038/ni.1849

15. Weimershaus M, Evnouchidou I, Saveanu L, Van Endert P. Peptidases trimming MHC class I ligands. Curr Opin Immunol (2013) 25:90-6. doi:10.1016/j.coi. 2012.10.001

16. Cascio P, Hilton C, Kisselev AF, Rock KL, Goldberg AL. 26S proteasomes and immunoproteasomes produce mainly $\mathrm{N}$-extended versions of an antigenic peptide. EMBO J (2001) 20:2357-66. doi:10.1093/emboj/20.10.2357

17. Serwold T, Gonzalez F, Kim J, Jacob R, Shastri N. ERAAP customizes peptides for MHC class I molecules in the endoplasmic reticulum. Nature (2002) 419:480-3. doi:10.1038/nature01074

18. Saveanu L, Carroll O, Lindo V, Del Val M, Lopez D, Lepelletier Y, et al. Concerted peptide trimming by human ERAP1 and ERAP2 aminopeptidase complexes in the endoplasmic reticulum. Nat Immunol (2005) 6:689-97. doi:10.1038/ni1208

19. Hulpke S, Tampe R. The MHC I loading complex: a multitasking machinery in adaptive immunity. Trends Biochem Sci (2013) 38:412-20. doi:10.1016/j.tibs. 2013.06.003

20. York IA, Chang SC, Saric T, Keys JA, Favreau JM, Goldberg AL, et al. The ER aminopeptidase ERAP1 enhances or limits antigen presentation by trimming epitopes to 8-9 residues. Nat Immunol (2002) 3:1177-84. doi:10.1038/ni860

21. Yan J, Parekh VV, Mendez-Fernandez Y, Olivares-Villagomez D, Dragovic S, Hill $\mathrm{T}$, et al. In vivo role of ER-associated peptidase activity in tailoring peptides for presentation by MHC class Ia and class Ib molecules. J Exp Med (2006) 203:647-59. doi:10.1084/jem.20052271

22. York IA, Brehm MA, Zendzian S, Towne CF, Rock KL. Endoplasmic reticulum aminopeptidase 1 (ERAP1) trims MHC class I-presented peptides in vivo and plays an important role in immunodominance. Proc Natl Acad Sci U S A (2006) 103:9202-7. doi:10.1073/pnas.0603095103

23. Georgiadou D, Stratikos E. Cellular mechanisms that edit the immunopeptidome. Curr Proteomics (2009) 6:13-24. doi:10.2174/157016409787847439

24. Rastall DP, Aldhamen YA, Seregin SS, Godbehere S, Amalfitano A. ERAP1 functions override the intrinsic selection of specific antigens as immunodominant peptides, thereby altering the potency of antigen-specific cytolytic and effector memory T-cell responses. Int Immunol (2014) 26(12):685-95. doi:10.1093/intimm/dxu078

25. Hammer GE, Gonzalez F, Champsaur M, Cado D, Shastri N. The aminopeptidase ERAAP shapes the peptide repertoire displayed by major histocompatibility complex class I molecules. Nat Immunol (2006) 7:103-12. doi:10.1038/ni1286

26. Hammer GE, Gonzalez F, James E, Nolla H, Shastri N. In the absence of aminopeptidase ERAAP, MHC class I molecules present many unstable and highly immunogenic peptides. Nat Immunol (2007) 8:101-8. doi:10.1038/ ni1409

27. Tanioka T, Hattori A, Masuda S, Nomura Y, Nakayama H, Mizutani S, et al. Human leukocyte-derived arginine aminopeptidase. The third member of the oxytocinase subfamily of aminopeptidases. J Biol Chem (2003) 278:32275-83. doi:10.1074/jbc.M305076200

28. Zervoudi E, Papakyriakou A, Georgiadou D, Evnouchidou I, Gajda A, Poreba $\mathrm{M}$, et al. Probing the S1 specificity pocket of the aminopeptidases that generate antigenic peptides. Biochem J (2011) 435:411-20. doi:10.1042/BJ20102049

29. Birtley JR, Saridakis E, Stratikos E, Mavridis IM. The crystal structure of human endoplasmic reticulum aminopeptidase 2 reveals the atomic basis for 
distinct roles in antigen processing. Biochemistry (2012) 51:286-95. doi:10.1021/ bi201230p

30. Evnouchidou I, Weimershaus M, Saveanu L, Van Endert P. ERAP1ERAP2 dimerization increases peptide-trimming efficiency. J Immunol (2014) 193:901-8. doi:10.4049/jimmunol.1302855

31. Cui X, Hawari F, Alsaaty S, Lawrence M, Combs CA, Geng W, et al. Identification of ARTS-1 as a novel TNFR1-binding protein that promotes TNFR1 ectodomain shedding. J Clin Invest (2002) 110:515-26. doi:10.1172/JCI200213847

32. Cui X, Rouhani FN, Hawari F, Levine SJ. An aminopeptidase, ARTS-1, is required for interleukin-6 receptor shedding. J Biol Chem (2003) 278:28677-85. doi:10.1074/jbc.M300456200

33. Cui X, Rouhani FN, Hawari F, Levine SJ. Shedding of the type II IL-1 decoy receptor requires a multifunctional aminopeptidase, aminopeptidase regulator of TNF receptor type 1 shedding. J Immunol (2003) 171:6814-9. doi:10.4049/jimmunol.171.12.6814

34. Goto Y, Ogawa K, Hattori A, Tsujimoto M. Secretion of endoplasmic reticulum aminopeptidase 1 is involved in the activation of macrophages induced by lipopolysaccharide and interferon-gamma. J Biol Chem (2011) 286:21906-14. doi:10.1074/jbc.M111.239111

35. Goto Y, Ogawa K, Nakamura TJ, Hattori A, Tsujimoto M. TLR-mediated secretion of endoplasmic reticulum aminopeptidase 1 from macrophages. JImmunol (2014) 192:4443-52. doi:10.4049/jimmunol.1300935

36. Aldhamen YA, Seregin SS, Rastall DP, Aylsworth CF, Pepelyayeva Y, Busuito CJ, et al. Endoplasmic reticulum aminopeptidase-1 functions regulate key aspects of the innate immune response. PLoS One (2013) 8:e69539. doi:10.1371/journal. pone. 0069539

37. Cifaldi L, Lo Monaco E, Forloni M, Giorda E, Lorenzi S, Petrini S, et al. Natural killer cells efficiently reject lymphoma silenced for the endoplasmic reticulum aminopeptidase associated with antigen processing. Cancer Res (2011) 71:1597-606. doi:10.1158/0008-5472.CAN-10-3326

38. James E, Bailey I, Sugiyarto G, Elliott T. Induction of Protective Antitumor Immunity through Attenuation of ERAAP Function. J Immunol (2013) 190:5839-46. doi:10.4049/jimmunol.1300220

39. Fruci D, Ferracuti S, Limongi MZ, Cunsolo V, Giorda E, Fraioli R, et al. Expression of endoplasmic reticulum aminopeptidases in EBV-B cell lines from healthy donors and in leukemia/lymphoma, carcinoma, and melanoma cell lines. J Immunol (2006) 176:4869-79. doi:10.4049/jimmunol.176.8.4869

40. Andres AM, Dennis MY, Kretzschmar WW, Cannons JL, Lee-Lin SQ, Hurle B, et al. Balancing selection maintains a form of ERAP2 that undergoes nonsensemediated decay and affects antigen presentation. PLoS Genet (2010) 6:e1001157. doi:10.1371/journal.pgen.1001157

41. Kamphausen E, Kellert C, Abbas T, Akkad N, Tenzer S, Pawelec G, et al. Distinct molecular mechanisms leading to deficient expression of ERresident aminopeptidases in melanoma. Cancer Immunol Immunother (2010) 59:1273-84. doi:10.1007/s00262-010-0856-7

42. Fruci D, Giacomini P, Nicotra MR, Forloni M, Fraioli R, Saveanu L, et al. Altered expression of endoplasmic reticulum aminopeptidases ERAP1 and ERAP2 in transformed non-lymphoid human tissues. J Cell Physiol (2008) 216:742-9. doi:10.1002/jcp. 21454

43. Stoehr CG, Buettner-Herold M, Kamphausen E, Bertz S, Hartmann A, Seliger B. Comparative expression profiling for human endoplasmic reticulum-resident aminopeptidases 1 and 2 in normal kidney versus distinct renal cell carcinoma subtypes. Int J Clin Exp Pathol (2013) 6:998-1008.

44. Mehta AM, Jordanova ES, Kenter GG, Ferrone S, Fleuren GJ. Association of antigen processing machinery and HLA class I defects with clinicopathological outcome in cervical carcinoma. Cancer Immunol Immunother (2008) 57:197-206. doi:10.1007/s00262-007-0362-8

45. Ayshamgul H, Ma H, Ilyar S, Zhang LW, Abulizi A. Association of defective HLA-I expression with antigen processing machinery and their association with clinicopathological characteristics in Kazak patients with esophageal cancer. Chin Med $J$ (Engl) (2011) 124(3):341-6. doi:10.3760/cma.j.issn.0366-6999.2011.03.004

46. Hasim A, Abudula M, Aimiduo R, Ma JQ, Jiao Z, Akula G, et al. Posttranscriptional and epigenetic regulation of antigen processing machinery (APM) components and HLA-I in cervical cancers from Uighur women. PLoS One (2012) 7:e44952. doi:10.1371/journal.pone.0044952

47. Burton PR, Clayton DG, Cardon LR, Craddock N, Deloukas P, Duncanson A, et al. Association scan of 14,500 nonsynonymous SNPs in four diseases identifies autoimmunity variants. Nat Genet (2007) 39:1329-37. doi:10.1038/ng.2007.17
48. Evans DM, Spencer CC, Pointon JJ, Su Z, Harvey D, Kochan G, et al. Interaction between ERAP1 and HLA-B27 in ankylosing spondylitis implicates peptide handling in the mechanism for HLA-B27 in disease susceptibility. Nat Genet (2011) 43:761-7. doi:10.1038/ng.873

49. International Genetics of Ankylosing Spondylitis Consortium (IGAS), Cortes A, Hadler J, Pointon JP, Robinson PC, Karaderi T, et al. Identification of multiple risk variants for ankylosing spondylitis through high-density genotyping of immune-related loci. Nat Genet (2013) 45:730-8. doi:10.1038/ng.2667

50. Szczypiorska M, Sanchez A, Bartolome N, Arteta D, Sanz J, Brito E, et al. ERAP1 polymorphisms and haplotypes are associated with ankylosing spondylitis susceptibility and functional severity in a Spanish population. Rheumatology (Oxford) (2011) 50:1969-75. doi:10.1093/rheumatology/ker229

51. Maksymowych WP, Inman RD, Gladman DD, Reeve JP, Pope A, Rahman P. Association of a specific ERAP1/ARTS1 haplotype with disease susceptibility in ankylosing spondylitis. Arthritis Rheum (2009) 60:1317-23. doi:10.1002/art.24467

52. Tsui FW, Haroon N, Reveille JD, Rahman P, Chiu B, Tsui HW, et al. Association of an ERAP1 ERAP2 haplotype with familial ankylosing spondylitis. Ann Rheum Dis (2010) 69:733-6. doi:10.1136/ard.2008.103804

53. Kirino Y, Bertsias G, Ishigatsubo Y, Mizuki N, Tugal-Tutkun I, Seyahi E, et al. Genome-wide association analysis identifies new susceptibility loci for Behcet's disease and epistasis between HLA-B ${ }^{\star} 51$ and ERAP1. Nat Genet (2013) 45:202-7. doi:10.1038/ng.2520

54. Ombrello MJ, Kirino Y, De Bakker PI, Gul A, Kastner DL, Remmers EF. Behcet disease-associated MHC class I residues implicate antigen binding and regulation of cell-mediated cytotoxicity. Proc Natl Acad Sci U S A (2014) 111:8867-72. doi:10.1073/pnas.1406575111

55. Strange A, Capon F, Spencer CC, Knight J, Weale ME, Allen MH, et al. A genome-wide association study identifies new psoriasis susceptibility loci and an interaction between HLA-C and ERAP1. Nat Genet (2010) 42:985-90. doi:10.1038/ng.694

56. Yamamoto N, Nakayama J, Yamakawa-Kobayashi K, Hamaguchi H, Miyazaki R, Arinami T. Identification of 33 polymorphisms in the adipocyte-derived leucine aminopeptidase (ALAP) gene and possible association with hypertension. Hum Mutat (2002) 19:251-7. doi:10.1002/humu.10047

57. Guerini FR, Cagliani R, Forni D, Agliardi C, Caputo D, Cassinotti A, et al. A functional variant in ERAP1 predisposes to multiple sclerosis. PLoS One (2012) 7:e29931. doi:10.1371/journal.pone.0029931

58. Johnson MP, Roten LT, Dyer TD, East CE, Forsmo S, Blangero J, et al. The ERAP2 gene is associated with preeclampsia in Australian and Norwegian populations. Hum Genet (2009) 126:655-66. doi:10.1007/s00439-009-0714-x

59. Cagliani R, Riva S, Biasin M, Fumagalli M, Pozzoli U, Lo Caputo S, et al. Genetic diversity at endoplasmic reticulum aminopeptidases is maintained by balancing selection and is associated with natural resistance to HIV-1 infection. Hum Mol Genet (2010) 19:4705-14. doi:10.1093/hmg/ddq401

60. Hill LD, Hilliard DD, York TP, Srinivas S, Kusanovic JP, Gomez R, et al. Fetal ERAP2 variation is associated with preeclampsia in African Americans in a casecontrol study. BMC Med Genet (2011) 12:64. doi:10.1186/1471-2350-12-64

61. Vanhille DL, Hill LD, Hilliard DD, Lee ED, Teves ME, Srinivas S, et al. A novel ERAP2 haplotype structure in a Chilean population: implications for ERAP2 protein expression and preeclampsia risk. Mol Genet Genomic Med (2013) 1:98-107. doi:10.1002/mgg3.13

62. Kuiper JJ, Van Setten J, Ripke S, Van't Slot R, Mulder F, Missotten T, et al. A genome-wide association study identifies a functional ERAP2 haplotype associated with birdshot chorioretinopathy. Hum Mol Genet (2014) 289(7):3978-90. doi:10.1093/hmg/ddu307

63. Goto Y, Hattori A, Ishii Y, Tsujimoto M. Reduced activity of the hypertensionassociated Lys528Arg mutant of human adipocyte-derived leucine aminopeptidase (A-LAP)/ER-aminopeptidase-1. FEBS Lett (2006) 580:1833-8. doi:10. 1016/j.febslet.2006.02.041

64. Evnouchidou I, Kamal RP, Seregin SS, Goto Y, Tsujimoto M, Hattori A, et al. Coding single nucleotide polymorphisms of endoplasmic reticulum aminopeptidase 1 can affect antigenic peptide generation in vitro by influencing basic enzymatic properties of the enzyme. J Immunol (2011) 186:1909-13. doi:10. 4049/jimmunol.1003337

65. Kochan G, Krojer T, Harvey D, Fischer R, Chen L, Vollmar M, et al. Crystal structures of the endoplasmic reticulum aminopeptidase-1 (ERAP1) reveal the molecular basis for N-terminal peptide trimming. Proc Natl Acad Sci U S A (2011) 108:7745-50. doi:10.1073/pnas.1101262108 
66. Martin-Esteban A, Gomez-Molina P, Sanz-Bravo A, Lopez De Castro JA. Combined effects of ankylosing spondylitis-associated ERAP1 polymorphisms outside the catalytic and peptide-binding sites on the processing of natural HLA-B27 ligands. J Biol Chem (2014) 289(7):3978-90. doi:10.1074/jbc.M113. 529610

67. Reeves E, Edwards CJ, Elliott T, James E. Naturally occurring ERAP1 haplotypes encode functionally distinct alleles with fine substrate specificity. J Immunol (2013) 191:35-43. doi:10.4049/jimmunol.1300598

68. Chen L, Fischer R, Peng Y, Reeves E, McHugh K, Ternette N, et al. Critical role of endoplasmic reticulum aminopeptidase 1 in determining the length and sequence of peptides bound and presented by HLA-B27. Arthritis Rheumatol (2014) 66:284-94. doi:10.1002/art.38249

69. Nguyen TT, Chang SC, Evnouchidou I, York IA, Zikos C, Rock KL, et al. Structural basis for antigenic peptide precursor processing by the endoplasmic reticulum aminopeptidase ERAP1. Nat Struct Mol Biol (2011) 18:604-13. doi: $10.1038 / \mathrm{nsmb} .2021$

70. Seregin SS, Rastall DP, Evnouchidou I, Aylsworth CF, Quiroga D, Kamal RP, et al. Endoplasmic reticulum aminopeptidase- 1 alleles associated with increased risk of ankylosing spondylitis reduce HLA-B27 mediated presentation of multiple antigens. Autoimmunity (2013) 46(8):497-508. doi:10.3109/08916934.2013. 819855

71. Reeves E, Colebatch A, Elliott T, Edwards C, James E. Functionally distinct ERAP1 allotype combinations distinguish individuals with Ankylosing Spondylitis. Proc Natl Acad Sci U S A (2014) 111(49):17594-9. doi:10.1073/ pnas. 1408882111

72. Garcia-Medel N, Sanz-Bravo A, Van Nguyen D, Galocha B, Gomez-Molina P, Martin-Esteban A, et al. Functional interaction of the ankylosing spondylitis associated endoplasmic reticulum aminopeptidase 1 polymorphism and HLAB27 in vivo. Mol Cell Proteomics (2012) 11(11):1416-29. doi:10.1074/mcp.M112. 019588

73. Garcia-Medel N, Sanz-Bravo A, Alvarez-Navarro C, Gomez-Molina P, Barnea E, Marcilla M, et al. Peptide handling by HLA-B27 subtypes influences their biological behavior, association with ankylosing spondylitis and susceptibility to ERAP1. Mol Cell Proteomics (2014) 13(12):3367-80. doi:10.1074/mcp.M114. $039214 \mathrm{~s}$
74. Evnouchidou I, Birtley J, Seregin S, Papakyriakou A, Zervoudi E, Samiotaki $\mathrm{M}$, et al. A common single nucleotide polymorphism in endoplasmic reticulum aminopeptidase 2 induces a specificity switch that leads to altered antigen processing. J Immunol (2012) 189:2383-92. doi:10.4049/jimmunol.1200918

75. Mehta AM, Jordanova ES, Van Wezel T, Uh HW, Corver WE, Kwappenberg KM, et al. Genetic variation of antigen processing machinery components and association with cervical carcinoma. Genes Chromosomes Cancer (2007) 46:577-86. doi:10.1002/gcc.20441

76. Mehta AM, Jordanova ES, Corver WE, Van Wezel T, Uh HW, Kenter GG, et al. Single nucleotide polymorphisms in antigen processing machinery component ERAP1 significantly associate with clinical outcome in cervical carcinoma. Genes Chromosomes Cancer (2009) 48:410-8. doi:10.1002/gcc.20648

77. Zervoudi E, Saridakis E, Birtley JR, Seregin SS, Reeves E, Kokkala P, et al. Rationally designed inhibitor targeting antigen-trimming aminopeptidases enhances antigen presentation and cytotoxic T-cell responses. Proc Natl Acad Sci U S A (2013) 110:19890-5. doi:10.1073/pnas.1309781110

Conflict of Interest Statement: The authors declare that the research was conducted in the absence of any commercial or financial relationships that could be construed as a potential conflict of interest.

Received: 20 October 2014; accepted: 28 November 2014; published online: 19 December 2014.

Citation: Stratikos E, Stamogiannos A, Zervoudi E and Fruci D (2014) A role for naturally occurring alleles of endoplasmic reticulum aminopeptidases in tumor immunity and cancer pre-disposition. Front. Oncol. 4:363. doi: 10.3389/fonc.2014.00363

This article was submitted to Tumor Immunity, a section of the journal Frontiers in Oncology.

Copyright (c) 2014 Stratikos, Stamogiannos, Zervoudi and Fruci. This is an open-access article distributed under the terms of the Creative Commons Attribution License (CC $B Y)$. The use, distribution or reproduction in other forums is permitted, provided the original author(s) or licensor are credited and that the original publication in this journal is cited, in accordance with accepted academic practice. No use, distribution or reproduction is permitted which does not comply with these terms. 